\title{
A FAMILY OF POLYNOMIALS WITH THE UNIQUENESS PROPERTY FOR LINEARLY NON-DEGENERATE HOLOMORPHIC MAPPINGS
}

\author{
ManABU ShIROSAKI
}

\section{§1. Introduction}

Let $\mathscr{F}$ be a family of nonconstant holomorphic mappings of $\boldsymbol{C}$ into $\boldsymbol{P}^{n}(\boldsymbol{C})$ and $P$ be a homogeneous polynomial of $n+1$ variables. We call $P$ has the uniqueness property for $\mathscr{F}$ if $P(\tilde{f}) \not \equiv$ for any $f \in \mathscr{F}$ and $P(\tilde{f})=\alpha P(\tilde{g})$ implies $f=g$ for any two elements $f$ and $g$ of $\mathscr{F}$, where $\tilde{f}$ and $\tilde{g}$ are reduced representations of $f$ and $g$, respectively, and $\alpha$ is an entire function without zeros. Such an example was first given by $\mathrm{Yi}$ for nonconstant entire functions in $[\mathrm{Y}]$, and it was a polynomial of one variable.

The author gave polynomials with the uniqueness property for algebraically non-degenerate holomorphic mappings in $[\mathrm{S} 1]$, and for linearly non-degenerate holomorphic mappings in [S2]. The former has several irreducible components, but the number of irreducible components does not depend on $n$, and it is not easy to judge the irreducibility of the latter.

In this paper, we give homogeneous polynomials with the uniqueness property for linearly non-degenerate holomorphic mappings, whose degrees are lower than that in [S2], and irreducible ones for example.

However the problem of the uniqueness of nonconstant holomorphic mappings is very difficult. The author has not yet found homogeneous polynomials with the uniqueness property for nonconstant holomorphic mappings.

\section{§2. Known results}

First, we introduce a useful theorem by Green and Fujimoto. We mean a nonzero entire function by an entire function with a point whose value is not zero. For two nonzero entire functions $f$ and $g$, we say that they are equivalent if the ratio $f / g$ is constant. This introduces an equivalence relation in each set of nonzero entire functions. The following lemma due to $\mathrm{H}$. Fujimoto and M. Green is the key lemma for our theorem (cf. [F, Corollary 6.4] and [G, p. 70]):

Keywords: Uniqueness theorem, value distribution theory.

Received February 4, 2002; revised May 24, 2002. 
A FAMILY OF POLYNOMIALS WITH THE UNIQUENESS PROPERTY

LemMa 2.1. Let $f_{0}, \ldots, f_{n}$ be nonzero entire functions such that $f_{0}^{d}+\cdots+$ $f_{n}^{d}=0$, where $d$ is a positive integer. If $d \geq n^{2}$, then

$$
\sum_{f_{j} \in I} f_{j}^{d}=0
$$

for each equivalence class I of $\left\{f_{0}, \ldots, f_{n}\right\}$. Especially each class has at least two elements.

Definition 2.2. Let $f$ be a holomorphic mapping of $\boldsymbol{C}$ into $\boldsymbol{P}^{n}(\boldsymbol{C})$. A representation $\boldsymbol{f}=\left(f_{0}, \ldots, f_{n}\right)$ of $f$ is a holomorphic mapping of $\boldsymbol{C}$ into $\boldsymbol{C}^{n+1}$ such that $\boldsymbol{f}^{-1}(\mathbf{0}) \neq \boldsymbol{C}$ and $f(z)=\left(f_{0}(z): \cdots: f_{n}(z)\right)$ for each $z \in \boldsymbol{C} \backslash \boldsymbol{f}^{-1}(\mathbf{0})$, where $\left(z_{0}: \cdots: z_{n}\right)$ is a homogeneous coordinate system. A representation $\boldsymbol{f}$ is called to be reduced if $\boldsymbol{f}^{-1}(\mathbf{0})=\emptyset$.

Definition 2.3. A holomorphic mapping $f$ of $\boldsymbol{C}$ into $\boldsymbol{P}^{n}(\boldsymbol{C})$ is linearly nondegenerate if its image is not contained in any hyperplane of $\boldsymbol{P}^{n}(\boldsymbol{C})$. This is equivalent to that $f_{0}, \ldots, f_{n}$ are linearly independent over $\boldsymbol{C}$, where $\left(f_{0}, \ldots, f_{n}\right)$ is a representation of $f$.

For a very special case of the result of $[\mathrm{S} 2]$, we introduce

THeOREM 2.4. For $d \geq(2 n-1)^{2}$, the polynomial

$$
\sum_{j=0}^{n-1}\left(z_{j}^{13}+z_{j}^{11} z_{j+1}^{2}+z_{j+1}^{13}\right)^{d}
$$

has the uniqueness property for linearly non-degenerate holomorphic mappings.

The least degree of the polynomials of the above theorem is $13(2 n-1)^{2}$.

\section{§3. Uniqueness of holomorphic mappings}

Let $\boldsymbol{v}_{j}(j=0, \ldots, q)$ be $q+1$ row vectors of $\boldsymbol{C}^{n+1}$, where $q \geq n+1$. Define the set $Q:=\left\{\alpha=\left(\alpha_{0}, \ldots, \alpha_{n}\right): \alpha_{0}, \ldots, \alpha_{n}\right.$ are distinct integers, $0 \leq \alpha_{0}, \ldots$, $\left.\alpha_{n} \leq q,\right\}$. For each element $\alpha=\left(\alpha_{0}, \ldots, \alpha_{n}\right) \in Q$, we put $\bar{\alpha}=\left\{\alpha_{0}, \ldots, \alpha_{n}\right\}$, and associate the matrix

$$
A_{\alpha}=\left(\begin{array}{c}
\boldsymbol{v}_{\alpha_{0}} \\
\vdots \\
\boldsymbol{v}_{\alpha_{n}}
\end{array}\right) .
$$

Take a positive integer $d$, and we assume:

(A1) $v_{j}(0 \leq j \leq q)$ are in general position;

(A2) take any $\alpha, \alpha^{\prime}, \beta, \beta^{\prime} \in Q$. If $\bar{\alpha} \neq \overline{\alpha^{\prime}}, \bar{\beta} \neq \overline{\beta^{\prime}}$, and if $\bar{\alpha} \neq \bar{\beta}$ or $\overline{\alpha^{\prime}} \neq \overline{\beta^{\prime}}$, then 


$$
\left(\frac{\operatorname{det} A_{\alpha}}{\operatorname{det} A_{\alpha^{\prime}}}\right)^{d} \neq\left(\frac{\operatorname{det} A_{\beta}}{\operatorname{det} A_{\beta^{\prime}}}\right)^{d} \text {. }
$$

For two vectors $\boldsymbol{z}=\left(z_{0}, \ldots, z_{n}\right)$ and $\boldsymbol{w}=\left(w_{0}, \ldots, w_{n}\right)$, we define $\boldsymbol{z} \cdot \boldsymbol{w}=$ $z_{0} w_{0}+\cdots+z_{n} w_{n}$. Let $\left(z_{0}: \cdots: z_{n}\right)$ be a homogeneous coordinate system of $\boldsymbol{P}^{n}(\boldsymbol{C})$ and write $\boldsymbol{z}=\left(z_{0}, \ldots, z_{n}\right)$. Define the homogeneous polynomial $P$ by $P\left(z_{0}, \ldots, z_{n}\right)=\sum_{j=0}^{q}\left(\boldsymbol{v}_{j} \cdot \boldsymbol{z}\right)^{d}=0$.

Then we have the following:

Theorem 3.1. Suppose $d \geq(2 q+1)^{2}$. Then, the polynomial $P$ has the uniqueness property for linearly non-degenerate holomorphic mappings of $\boldsymbol{C}$ into $\boldsymbol{P}^{n}(\boldsymbol{C})$, i.e., for linearly non-degenerate holomorphic mappings $f$ and $g$ of $\boldsymbol{C}$ into $\boldsymbol{P}^{n}(\boldsymbol{C})$ with reduced representations $\boldsymbol{f}$ and $\boldsymbol{g}$, respectively,

$$
\sum_{j=0}^{q}\left(\boldsymbol{v}_{j} \cdot \boldsymbol{f}\right)^{d}=\varphi \sum_{j=0}^{q}\left(\boldsymbol{v}_{j} \cdot \boldsymbol{g}\right)^{d}
$$

for an entire function $\varphi$ without zeros implies $f=g$.

Proof. At the beginning of the proof, we note that none of $\boldsymbol{v}_{j} \cdot \boldsymbol{f}, \boldsymbol{v}_{j} \cdot \boldsymbol{g}$ is identically equal to zero by linear non-degeneracy of $f$ and $g$ and we may assume that $\varphi \equiv 1$ by changing reduced representations.

We apply Lemma 2.1 to (1) considering linear non-degeneracy of $f$ and $g$. Then there exists a permutation $\sigma$ of $0, \ldots, q$ and $d$-th roots $\omega_{0}, \ldots, \omega_{q}$ of 1 such that

$$
\boldsymbol{v}_{j} \cdot \boldsymbol{f}=\omega_{j} \boldsymbol{v}_{\sigma(j)} \cdot \boldsymbol{g} \quad(0 \leq j \leq q) .
$$

For each arbitrary $\alpha \in Q$, we get by (2)

$$
A_{\alpha}{ }^{t} \boldsymbol{f}=\Omega_{\alpha} A_{\sigma(\alpha)}{ }^{t} \boldsymbol{g},
$$

where $\sigma(\alpha)=\left(\sigma\left(\alpha_{0}\right), \ldots, \sigma\left(\alpha_{n}\right)\right) \in Q$ and

$$
\Omega_{\alpha}=\left(\begin{array}{ccc}
\omega_{\alpha_{0}} & & 0 \\
& \ddots & \\
0 & & \omega_{\alpha_{n}}
\end{array}\right) .
$$

Take $\alpha, \beta \in Q$ with $\bar{\alpha} \neq \bar{\beta}$. By deleting ${ }^{t} \boldsymbol{f}$ from the equation (3) and its correspondence for $\beta$, we get $A_{\beta} A_{\alpha}^{-1} \Omega_{\alpha} A_{\sigma(\alpha)}{ }^{t} \boldsymbol{g}=\Omega_{\beta} A_{\sigma(\beta)}{ }^{t} \boldsymbol{g}$. By linear nondegeneracy of $g$ we have

$$
A_{\beta} A_{\alpha}^{-1} \Omega_{\alpha} A_{\sigma(\alpha)}=\Omega_{\beta} A_{\sigma(\beta)} ;
$$

thus, $A_{\alpha}^{-1} \Omega_{\alpha} A_{\sigma(\alpha)}=A_{\beta}^{-1} \Omega_{\beta} A_{\sigma(\beta)}$. By taking $d$-th powers of determinants of both sides, $\left(\operatorname{det} A_{\beta} / \operatorname{det} A_{\alpha}\right)^{d}=\left(\operatorname{det} A_{\sigma(\beta)} / \operatorname{det} A_{\sigma(\alpha)}\right)^{d}$. The assumption (A2) requires $\bar{\alpha}=\overline{\sigma(\alpha)}, \bar{\beta}=\overline{\sigma(\beta)}$, which induce that $\sigma$ is the identity. Hence, we have, by (3), 


$$
A^{t} \boldsymbol{f}=\Omega A^{t} \boldsymbol{g}
$$

where $A=A_{(0,1, \ldots, n)}$ and $\Omega=\Omega_{(0,1, \ldots, n)}$. The equation (2) for $j=n+1$ is $\boldsymbol{v}_{n+1} \cdot \boldsymbol{f}=\omega_{n+1} \boldsymbol{v}_{n+1} \cdot \boldsymbol{g}$. By deleting $\boldsymbol{f}$ from this and (4), $\boldsymbol{v}_{n+1} A^{-1} \boldsymbol{\Omega} A^{t} \boldsymbol{g}=$ $\omega_{n+1} \boldsymbol{v}_{n+1}{ }^{t} \boldsymbol{g}$ is obtained. Since $g$ is linearly non-degenerate, $\boldsymbol{v}_{n+1} A^{-1} \Omega A=$ $\omega_{n+1} \boldsymbol{v}_{n+1}$, and hence,

$$
\boldsymbol{v}_{n+1} A^{-1} \Omega=\omega_{n+1} \boldsymbol{v}_{n+1} A^{-1} .
$$

By the assumption (A1), there exist nonzero constants $a_{0}, \ldots, a_{n}$ such that

$$
\boldsymbol{v}_{n+1}=a_{0} \boldsymbol{v}_{0}+\cdots+a_{n} \boldsymbol{v}_{n} .
$$

Then, $\boldsymbol{v}_{n+1} A^{-1}=\left(a_{0}, \ldots, a_{n}\right)$. Therefore, we can get from $(5)\left(\omega_{0} a_{0}, \ldots, \omega_{n} a_{n}\right)=$ $\omega_{n+1}\left(a_{0}, \ldots, a_{n}\right)$, and thus $\omega_{0}=\cdots=\omega_{n}=\omega_{n+1}$, which implies $f=g$ by (4).

Q.E.D.

Remark 3.2. From (1), we can induce $\boldsymbol{f}=\psi \boldsymbol{g}$, where $\psi$ is an entire function such that $\psi^{d}=\varphi$.

Example 3.3. Here, we give an example for the above theorem.

Let $\quad \boldsymbol{v}_{0}=(1,0, \ldots, 0), \quad \boldsymbol{v}_{1}=(0,1,0, \ldots, 0), \ldots, \boldsymbol{v}_{n}=(0, \ldots, 0,1), \quad \boldsymbol{v}_{n+1}=$ $\left(a_{0}, \ldots, a_{n}\right)$. The condition (A1) is equivalent to $a_{j} \neq 0(0 \leq j \leq n)$. Since $\operatorname{det} A_{(0, \ldots, j-1, j+1, \ldots, n+1)}=(-1)^{n-j} a_{j}(0 \leq j \leq n)$ and $\operatorname{det} A_{(0, \ldots, n)}=1$, (A2) is equivalent to the condition that

$$
(-1)^{d(k-j)}\left(a_{j} / a_{k}\right)^{d} \neq(-1)^{d(v-\mu)}\left(a_{\mu} / a_{v}\right)^{d}
$$

for all $0 \leq j, k, \mu, v \leq n+1$ such that $j \neq k, \mu \neq v$ and $(j, k) \neq(\mu, v)$, where $a_{n+1}=-1$. If $a_{0}, \ldots, a_{n}$ satisfies the above conditions, the homogeneous polynomial

$$
z_{0}^{d}+\cdots+z_{n}^{d}+\left(a_{0} z_{0}+\cdots+a_{n} z_{n}\right)^{d}
$$

has the property of Theorem 3.1 for $d \geq(2 n+3)^{2}$.

Now, we consider the non-singularity of the hypersurface defined by the zero set of the polynomial. It is non-singular if and only if the equations $z_{j}^{d-1}+a_{j}\left(a_{0} z_{0}+\cdots+a_{n} z_{n}\right)^{d-1}=0(0 \leq j \leq n)$ have no common solution except for $(0, \ldots, 0)$, and it is fulfilled if

$$
\left|\begin{array}{cccc}
\eta_{0} a_{0}-1 & \eta_{0} a_{1} & \cdots & \eta_{0} a_{n} \\
\eta_{1} a_{0} & \eta_{1} a_{1}-1 & \cdots & \eta_{1} a_{n} \\
\vdots & \vdots & \ddots & \vdots \\
\eta_{n} a_{0} & \eta_{n} a_{1} & \cdots & \eta_{n} a_{n}-1
\end{array}\right| \neq 0
$$

for any $(d-1)$-th roots $\eta_{0}, \ldots, \eta_{n}$ of $-a_{0}, \ldots,-a_{n}$, respectively. Hence, if

$$
\eta_{0} a_{0}+\cdots+\eta_{n} a_{n} \neq 1
$$


for any $(d-1)$-th roots $\eta_{0}, \ldots, \eta_{n}$ of $-a_{0}, \ldots,-a_{n}$, respectively, the considering hypersurface is non-singular. For more explicit example, let $3=p_{1}<\cdots<p_{n}$ be prime numbers and $p_{0}=1$. Put $a_{j}=1 /\left(2^{j+1} p_{j}\right)(0 \leq j \leq n)$, then all above conditions are satisfied. Also, for $n \geq 2$ the least degree $(2 n+3)^{2}$ of the polynomial is smaller than those of previous results.

\section{REFERENCES}

[F] H. Fujimoto, On meromorphic maps into the complex projective space, J. Math. Soc. Japan, 26 (1974), 272-288.

[G] M. L. GReEN, Some Picard theorems for holomorphic maps to algebraic varieties, Amer. J. Math., 97 (1975), 43-75.

[S1] M. ShIRosaki, On polynomials which determine holomorphic mappings, J. Math. Soc. Japan, 49 (1997), 289-298.

[S2] M. ShIRosaki, A hypersurface which determines linearly non-degenerate holomorphic mappings, Kodai Math. J., 23 (2000), 105-107.

[Y] H.-X. YI, A question of Gross and the uniqueness of entire functions, Nagoya Math. J., 138 (1995), 169-177.

Department of Mathematical Sciences

College of ENGINEERING

Osaka Prefecture University

SAKAI, 599-8531 JAPAN

e-mail: mshiro@ms.osakafu-u.ac.jp 